\title{
FIT: An iterative parameter-estimation function in LISP
}

\author{
F. MICHAEL RABINOWITZ, MALCOLM J. GRANT, and H. LOUIS DINGLEY \\ Memorial University, St. John's, Newfoundland, Canada
}

\begin{abstract}
A nested parameter-estimation program (FIT), written in LISP, is described. FIT searches an n-dimensional parameter space for the set of parameter values associated with either a minimum or a maximum criterion score. The range of the parameters is cut in half on each iteration. Two versions of FIT, one suitable for use on large, mainframe systems and the other designed for small microprocessors, appear in the appendices.
\end{abstract}

A critical stage in the development of any model involves the estimation of free parameters. Typically, the goal of researchers is to find values for the parameters that maximize the goodness of fit between the model and some empirical data set. If the functional relationships between the goodness-of-fit criterion score and the various parameters can be specified mathematically, researchers can rely on well-established techniques for finding the optimal parameter values (Restle \& Greeno, 1970). If the relationships cannot be specified mathematically, researchers often have to rely on optimization methods involving a computer search of the parameter space (Wickens, 1982).

We have devised an iterative parameter-estimation program (FIT) to deal with the situations to which mathematical methods cannot easily be applied. The impetus for this program stemmed from our attempt to simulate the way in which people learn to play the counter game Nim. Three parameters were built into the simulation in an attempt to account for individual differences, and we needed an optimization procedure for estimating the parameter values for each subject.

Compared with many other optimization programs (see Daniels, 1978), FIT has some unique advantages. First, it is written in LISP, a programming language preferred by many researchers in both artificial intelligence and cognitive psychology (Simon, 1979; Winston \& Horn, 1981). Second, FIT works with any number of parameters. Dimension statements and terminal, loop index values are not used. Finally, FIT can be applied to any mathematical or computer model that generates a goodness-of-fit criterion score that is continuous over the parameter space. In view of these advantages, we believe FIT may be of considerable use to researchers in general, and especially to those developing models in the areas of artificial intelligence and cognitive psychology.

This research was supported by NSERC Grant A0304. Reprints are available from any of the authors at the Department of Psychology, Memorial University, St. John's, Newfoundland, Canada A1B $3 \times 9$

\section{DESCRIPTION}

FIT consists of four LISP functions, an executive function (startfit), a parameter-setting function (fit1), a score-optimizing function (fit2), and a numeric-mode function (mode).

\section{Executive Function}

The 18-line executive function assigns a middle value and a range to each parameter and calls the other three functions. With each iteration, the range of each parameter is cut in half and the search for the best parameter values continues within progressively narrower boundaries. When the range for each parameter narrows to a preset minimum value, further variations in that parameter are discontinued. When all parameter ranges have reached their respective minimum values, the executive function returns the final parameter values, together with the associated criterion score. Alternatively, the executive function can be modified to terminate when the criterion score reaches a value that is deemed to be good enough.

\section{Parameter-Setting Function}

The 40-line parameter-setting function takes the middle value and two boundary values for each parameter and creates a list of all $3^{n}$ possible combinations of parameter settings (where $\mathbf{n}=$ the number of parameters).

\section{Score-Optimizing Function}

The 15-line score-optimizing function computes a criterion score for each combination of parameter values using some function external to FIT. The nature of this external function will, of course, be dictated by the model being tested. The maximum or minimum criterion score and the associated parameter values are then returned to the executive function.

\section{Numeric-Mode Function}

The three-line numeric-mode function causes numerical operations to be carried out using either integer or floating-point arithmetic, depending on the wish of the user. 


\section{INPUT}

All of the information needed by FIT is entered in the argument list of the executive function. The first argument is a list containing the names of the parameters. The second argument is a quoted atom, either 'integer or 'float, depending on the desired arithmetic mode for the parameters. The third and fourth arguments are lists containing, respectively, the lower and upper boundaries for each of the parameters. The fifth argument is a list of cutoff values that specify the points at which further subdivision of the intervals around the best estimates of the parameters ceases. The sixth argument is the name of some LISP function that uses the parameters listed in the first argument of the executive function and returns some criterion value based upon the model being tested. Finally, the seventh argument in the executive function is a quoted atom, either 'min or 'max, indicating whether FIT is to search for parameter values associated with the minimum or maximum value of the criterion score.

\section{OUTPUT}

The output from FIT consists of a single list containing the minimum or maximum value of the criterion score followed by a list of the values of the various parameters that produced that criterion value.

\section{EXAMPLES}

The following examples illustrate how FIT is used. In the first example, the function demofit, which simply adds together the values of a list of parameters, is defined. Demofit is particularly useful because the number and range of parameters to which the function is applicable can be varied systematically without having a significant impact on the nearly instantaneous time in which demofit executes. On the other hand, the number of parameters determines the number of combinations of parameters FIT must generate on each iteration, and the range of the parameters and the cutoff values determine the number of iterations FIT must perform. Therefore, run-time estimates obtained when demofit is called by FIT are an accurate reflection of the time FIT takes to execute as a function of number and range of parameters on a particular computer. A variety of runtime estimates appear in Table 1. Demofit is defined as:

(defun demofit ()

(apply 'plus paramlist))

To find the maximum of this function when each of three parameters can range from 1 to 5 , FIT is called in the following way:

(startfit '(p1 $\mathrm{p} 2$ p3) 'integer '(1 1 1 1 ) '(5 5 (5

'(1 111$)$ 'demofit 'max)
The function returns

(12 (4 4 4))

Note that in integer arithmetic, FIT is unable to get within 1 of the parameter boundaries. If the same function is called using floating-point arithmetic, the values specified in the cutoff list determine how closely FIT can approach the parameter boundaries.

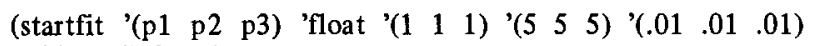
'demofit 'max)

The function returns

(14.9765625 (4.9921875 4.9921875 4.9921875))

In the next example, FIT is applied to the sine function, a system-defined function in Franz Lisp.

(startfit '(angle-in-radians) 'float '(0) '(6.28) '(.01) 'sin 'max)

The function returns

$(.99999(1.57))$

indicating that the sine function on the interval from 0 to $6.28(2 \pi)$ has a maximum of .99999 when angle $=$ 1.57 (or $\pi / 2$ ) radians.

Like most iterative fit procedures, FIT assumes some linear regularity and independence in the relationships between parameters and criterion score. Local minimums or maximums can pose a problem in situations in which these assumptions are violated. This problem is illustrated in the next example, in which a least-squares, line-fitting function was applied to a set of 18 paired scores. Standard regression techniques indicate that the best-fitting straight line for these data has a slope of +.67 and an intercept of -11.25 .

(startfit '(slope intercept) 'float '(-1 -20) '(1 20) '(.01 .01) 'linefit ' $\mathrm{min}$ )

returns

(187.44 (0.50 7.95))

Repeating the process with narrower starting boundaries results in a somewhat more accurate outcome.

(startfit '(slope intercept) 'float '(0 -15$)$ '(1 0$)^{\prime}$ '(.01 .01$)$
'linefit 'min)

returns

$(148.09(.65-8.91))$

Since the existence of local minimums and maximums is rarely known in advance, we recommend that research- 
Table 1

Run Times in Seconds for Demofit in Integer Mode

\begin{tabular}{|c|c|c|c|c|c|c|}
\hline \multirow[b]{2}{*}{ Computer } & \multirow[b]{2}{*}{ Language } & \multicolumn{2}{|c|}{ Parameters } & \multirow{2}{*}{$\begin{array}{l}\text { No. of } \\
\text { Parameter } \\
\text { Sets per } \\
\text { Iteration }\end{array}$} & \multirow{2}{*}{$\begin{array}{c}\text { No. of } \\
\text { Iterations }\end{array}$} & \multirow{2}{*}{$\begin{array}{c}\text { CPU } \\
\text { Run Time }\end{array}$} \\
\hline & & Number & Range & & & \\
\hline \multirow[t]{10}{*}{ VAX $11 / 780$} & Franz Lisp (Interpreted) & 1 & $1-16$ & 3 & 3 & 0.08 \\
\hline & & & $1-64$ & 3 & 5 & 0.15 \\
\hline & & 2 & $1-16$ & 9 & 3 & 0.26 \\
\hline & & & $1-64$ & 9 & 5 & 0.48 \\
\hline & & 3 & $1-16$ & 27 & 3 & 0.71 \\
\hline & & & $1-64$ & 27 & 5 & 1.55 \\
\hline & & 4 & $1-16$ & 81 & 3 & 2.60 \\
\hline & & & $1-64$ & 81 & 5 & 5.03 \\
\hline & & 5 & $1-16$ & 243 & 3 & 7.93 \\
\hline & & & $1-64$ & 243 & 5 & 15.64 \\
\hline \multirow[t]{10}{*}{ VAX $11 / 780$} & Franz Lisp (Compiled) & 1 & $1-16$ & 3 & 3 & 0.04 \\
\hline & & & $1-64$ & 3 & 5 & 0.05 \\
\hline & & 2 & $1-16$ & 9 & 3 & 0.08 \\
\hline & & & $1-64$ & 9 & 5 & 0.14 \\
\hline & & 3 & $1-16$ & 27 & 3 & 0.21 \\
\hline & & & $1-64$ & 27 & 5 & 0.62 \\
\hline & & 4 & $1-16$ & 81 & 3 & 0.75 \\
\hline & & & $1-64$ & 81 & 5 & 1.87 \\
\hline & & 5 & $1-16$ & 243 & 3 & 3.29 \\
\hline & & & $1-64$ & 243 & 5 & 6.42 \\
\hline \multirow[t]{4}{*}{ Sorcerer } & Stiff Upper Lisp & 1 & $1-16$ & 3 & 3 & \\
\hline & & & $1-64$ & 3 & 5 & 4.54 \\
\hline & & 2 & $1-16$ & 9 & 3 & 8.19 \\
\hline & & & $1-64$ & 9 & 5 & 15.04 \\
\hline
\end{tabular}

ers routinely repeat the fitting process several times, each time with a different set of starting boundaries. In this way, a familiarity with the contours of the parameter space can be obtained and the risk of being misled by local perturbations can be reduced. Wickens (1982) made essentially the same recommendation.

\section{LANGUAGE AND COMPUTER}

FIT is written in LISP and runs on a VAX 11/780 in a UNIX operating environment (see Appendix A). The version of LISP used ("Franz Lisp, Opus 38.79") was developed at the University of California at Berkeley and closely resembles MACLISP, the language developed at the Massachusetts Institute of Technology. In order to determine if the program could be used with LISP on a small system, the program was modified to run in integer mode using "Stiff Upper Lisp" on a 55K Z-80 Exidy Sorcerer computer (see Appendix B). This was a fairly conservative test because, in Stiff Upper Lisp, only integer arithmetic functions are available and the mapping functions are limited in both availability and scope.
In addition, the amount of memory available and the processing capacities of the Z-80 chip do not compare favorably with those of more recent microprocessors. Despite these limitations, FIT ran successfully on the Sorcerer, although, with more than two parameters, a stack-overflow problem was encountered. On the VAX, this problem occurred with more than five parameters. Run times on the VAX and Sorcerer computers for the demofit program over various parameter spaces appear in Table 1.

\section{REFERENCES}

DANIELs, R. W. (1978). An introduction to numerical methods and optimization techniques. New York: Elsevier-North Holland.

Restle, F., \& Greeno, J. G. (1970). Introduction to mathematical psychology. Reading, MA: Addison-Wesley.

Simon, H. A. (1979). Information processing models of cognition. Annual Review of Psychology, 30, 363-396.

Wickens, T. D. (1982). Models for behavior: Stochastic processes in psychology. San Francisco: Freeman.

Winston, P. H., \& Honn, B. K. P. (1981). LISP. Reading, MA: Addison-Wesley.

Appendix A

Franz Lisp Version of FIT

(defun startfit (nameset integer-float lower upper cutoff fname minmax)
(setq middlevalue (mapcar '(lambda (x5 y5) (quotient (plus x5 y5) (mode 2)))
lower upper)) 
(setq halfrange (mapcar '(lambda ( $x 5$ y5) (quotient (difference y5 x5)

lower upper))

(mode 2)))

(setq 11 (fitl middlevalue halfrange)); first call of fitl

(prog ()

loop

(cond ((equal (length 11) 1) (return (cons maxval 11)));iteration

; finished

(t (setq $r 1^{\prime}$ () tri (fit2 11) maxval (car tri) val (cdr tri))

; fit2 returns maximum score and parameter set

; $r l$ hold estimates of $\mathrm{fit}$ and associated parameter sets

(setq 11 (fitl val range1)); fitl returns a list of all

$($ go loop)))))

; possible parameter sets for the ; next iteration

(defun mode (i-f)

(cond ((equal integer-float 'float) (float $i-f)$ )

$(t i-f)))$

(defun fitl ( 11 12); middle values and half ranges respectively

(setq val (reverse 11) range '()); val is reverse so that last parameter

(prog ()

; is dealt with first

( setq rangel (mapcar

' (lambda (x y) (cond ( (lessp x y) ( $\operatorname{setq} x 0))$

12 cutoff)); new halfrange

$(t($ quotient $x(\operatorname{mode} 2)))))$

(setq range (reverse rangel)); reverse so that last parameter occurs ; first

(cond ((zerop (car range)) (setq 11 (list (1ist (car val))))); if the ; halfrange "hr" is zero then just list the parameter "p"

( $t$ (setq 11 (1ist (list (difference (car val) (car range))) (list (car val))

(list (plus (car val) (car range))))))) ; if

; halfrange not zero then list $\mathrm{p}-\mathrm{hr} \mathrm{p}$ and $\mathrm{p}+\mathrm{hr}$

( $s e t q$ val (cdr val) range (cdr range)); shorten the lists by one

( setq 13 (reverse 11) $14^{\prime}()$ ); 13 is reverse working set of 11

; 14 is temporary storage of 11

loopl; to consider second and remaining parameters

(cond ((null val) (return 11)); parameters exhausted return list of

((nu11 13) (go loop3)); 13 exhausted ; all possible combinations

(t (cond ( zerop (car range))

(

; list parameter

(t (setq workset (list (plus (car val) (car range))

(car val); list $\mathrm{p}-\mathrm{hr} \mathrm{p}$ p+hr

(difference (car val)

(car range)) )) ))) )

$100 \mathrm{p} 2$

(cond ((null workset) (setq $13(\operatorname{cdr} 13))$ (go loop1)); if workset empty ; shorten 13

(t (setq 14 (cons (cons (car workset) (car 13)) 14)); every value ; in the workset is paired with first value in $13-14$ has ; parameters stored in the original order 
(setq workset (cdr workset)) (go loop2))); shorten workset loop3; reset 13 and 11 - shorten val and range

(setq 13 (reverse 14) $1114 \mathrm{val}$ (cdr val) range (cdr range) $14^{\prime}($ )) (go $100 \mathrm{pl}))$ )

(defun fit2 (11)

(cond ( (null 11); 11 exhausted

(setq m (apply minmax (mapcar' (lambda $(x)(\operatorname{car} x)$ ) $r l))$ ); find

(setq val (car (mapcan'( lambda ( $x$ ) ; best fit

(cond ((equal ( $\operatorname{car} x) m$ )

(list $x)$ )

$((\mathrm{ni} 1))) \mathrm{r}())))$; select

; best fit and associated parameter set and store in val

( $t$ (

; names and values

(mapcar'set namelist paramlist); set names to values

(setq $r l$ (cons (cons (funcall fname) (car 11)) rl)); store fit and ; associated parameters in $\mathrm{rl}$

$($ fit2 $(\operatorname{cdr} 11)))))$; repeat with next parameter set

Auxiliary Functions

(defun demofit (); adds elements of a parameter list together

(apply 'plus paramlist))

(defun linefit (); sum of squared deviations about a line

(setq yprime (mapcar '(lambda ( $x 1)$ (plus (times slope $x 1$ ) intercept)) $x$ ))

(apply 'plus (mapcar

'(lambda (y2 y3) (times (difference y2 y3) (difference y2 y3)))

y yprime) ))

( $\operatorname{set} q \mathrm{x}$; data used by linefit

'(lll $\left.\begin{array}{llllllllllllllllll}118 & 99 & 118 & 121 & 123 & 98 & 131 & 121 & 108 & 111 & 118 & 112 & 113 & 111 & 106 & 102 & 113 & 101\end{array}\right)$

(setq y; data used by linefit

'( (6 $\left.\begin{array}{llllllllllllllllll}66 & 50 & 73 & 69 & 72 & 54 & 74 & 70 & 65 & 62 & 65 & 63 & 67 & 59 & 60 & 59 & 70 & 57\end{array}\right)$

\section{Appendix B \\ Stiff Upper Lisp Version of FIT}

(de startfit (nameset lower upper cutoff fname minmax)

( setq middlevalue (mapcar2 (lambda (x5 y5) (// ( + x5 y5) 2))

lower upper))

(setq halfrange (mapcar2 '(lambda (x5 y5) (// (- y5 x5) 2))

lower upper))

(setq 11 (fitl middlevalue halfrange)); first call of fit

(prog ()

loop

(cond ((equal (length 11) 1) (return (cons maxval 11))); iteration

; ; finished

(t (setq rl '() tri (fit2 11) maxval (car tri) val (cdr tri))

;; fit2 returns maximum score and parameter set

;; rl holds estimates of $\mathrm{fit}$ and associated parameter sets 
(setq 11 (fitl val rangel));; fitl returns a list of all ; ; possible parameter sets for

$(g \circ(0 \circ p)))))$

(de fitl (11 12); middle values and half ranges respectively

(setq val (reverse 11) range '()); val is reverse so that last parameter

(prog () ;; is dealt with first

(setq range1 (mapcar2

$$
\begin{array}{r}
(\text { lambda }(x y) \text { (cond }((<x y) \\
(\operatorname{setq} x))) \\
(t(/ / x 2))))
\end{array}
$$

12 cutoff)); new halfrange

(setq range (reverse range1)); reverse so that last parameter occurs ; ; first

(cond ((zerop (car range)) (setq 11 (1ist (list (car val))))); if the ; ; halfrange "hr" is zero then just list the parameter "p"

(t (setq 11 (list (list (- (car val) (car range)))

(list (car val))

(list $(+($ car val) (car range) $))))))$; ; if

; ; halfrange not zero then list $\mathrm{p}-\mathrm{hr} \mathrm{p}$ and $\mathrm{p}$ thr

(

( setq 13 (reverse 11) $14^{\prime}()$ );; 13 is reverse working set of 11

;; 14 is temporary storage of 11

loopl; ; to consider second and remaining parameters

(cond ((null val) (return 11)); ; parameters exhausted return list of ;; all possible combinations

((null 13) (go loop3));; 13 exhausted

( $t$ (cond ((zerop (car range))

( setq workset (list (car val)))); if halfrange zero

;; list parameter

(t (setq workset (list ( + (car val) (car range))

(car val);; 11st $\mathrm{p}$-her $\mathrm{p}$ pthr

(- (car val)

$100 \mathrm{p} 2$

$($ (car range $))))$ ))) )

(cond ((null workset) (setq $13(\operatorname{cdr} 13))$ (go loopl));; if workset empty ;; shorten 13

(t (setq 14 (cons (cons (car workset) (car 13)) 14)); ; every

; ; value in the workset is paired with first value in 13

;; 14 has parameters stored in the original order (setq workset (cdr workset)) (go loop2))); ; shorten workset

loop 3; reset 13 and 11 - shorten val and range

(setq 13 (reverse 14) $1114 \mathrm{val}$ (cdr val) range (cdr range) $14^{\prime}()$ )

(go loop1)))

(de fit2 (11)

(cond ( null 11); ; 11 exhausted

(setq m (apply minmax (mapcar '(lambda (x) (car x)) r1))); f find

(setq val (car (mapcanl' (lambda (x)

; ; best fit

$$
\begin{aligned}
&(\text { cond }((\text { equal }(\operatorname{car} x) m) \\
&(\text { list } x)) \\
&((\operatorname{nil}))) \mathrm{r} 1)))) ; \text {; select }
\end{aligned}
$$


; ; best fit and associated parameter set and store in val

( $t$ ( ; ; and values (mapcar2 'set namelist paramlist); ; set names to values ( $\operatorname{setq}$ r1 (cons (cons (apply fname) ( $\operatorname{car} 11)$ ) rl)); ; store fit and ; ; associated parameters in $\mathrm{rl}$ $($ fit2 $(\operatorname{cdr} 11)))))$; repeat with next parameter set

Auxiliary Functions

(de demofit (); adds elements of a parameter 1ist together (apply'+ paramlist))

(de linefit2 (); s sum of absolute deviations about a line (setq yprime (mapcar '(lambda $(x 1)(+(*$ slope $x 1)$ intercept)) $x)$ ) (apply'+ (mapcar2

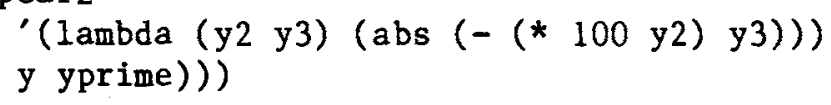

(setq $x ;$; data used by 1 inefit 2

'(ll8 $\left.\begin{array}{lllllllllllllllll}1189 & 118 & 121 & 123 & 98 & 131 & 121 & 108 & 111 & 118 & 112 & 113 & 111 & 106 & 102 & 113 & 101\end{array}\right)$

(setq y; d data used by linefit2

'( (66 $50 \quad 73 \quad 6972 \quad 5474 \quad 70 \quad 65 \quad 62 \quad 65 \quad 63 \quad 67 \quad 5960 \quad 59 \quad 70 \quad 57)$ )

(de mapcan1 (fname lst1); apply function fname to each element of 1stl

(apply 'append (mapcar fname lstl)))

(de abs ( $x$ ); ; returns absolute value of $x$

(cond $((<x 0)(*-1 x))$

$(t \quad x)))$

(de mapcar2 (fname 1st1 1st2); apply function fname to pairs of elements

(cond ( null lst 1$\left.)^{\prime}()\right)$ ; ; drawn from 1 st 1 and 1 st 2

( $t$ (cons (apply fname (1ist (car 1stl) (car 1st2)))

(mapcar2 fname (cdr 1st1) (cdr 1st2))))) )

(de zerop ( $x$ ); ; returns true if $x=0$

(cond ( equal $x 0) t$ )

$(t$ nil $))$ )

(de $\max \mathrm{n}$; ; returns the largest number in a set of numbers $(\max 1(\operatorname{car}($ list $n))))$

(de maxl (1st); ; returns the largest number in a list

(cond ( (equal (length 1st) l) (car 1st))

$((>(\operatorname{car} 1 s t)(\operatorname{set} q \operatorname{maximum}(\max 1(\operatorname{cdr} 1 s t))))$

(car 1st))

( $($ maximum $))$ )

(de min $n$; ; returns the smallest number in a set of numbers $(\operatorname{minl}(\operatorname{car}($ list $n))))$ 
314 RABINOWITZ, GRANT, AND DINGLEY

(de minl (1st); ; returns the smallest number in a list

(cond ((equal (length 1st) 1). (car 1st))

$((<(\operatorname{car} 1 s t)(\operatorname{setq}$ minimum $(\operatorname{minl}(\operatorname{cdr} 1 s t))))$

(car 1st))

(t minimum)))

(Manuscript received March 21, 1984;

revision accepted for publication May 15, 1984.) 